\title{
Control of Spin Dynamics of Excitons in Nanodots for Quantum Operations
}

\author{
Pochung Chen, C. Piermarocchi, and L. J. Sham \\ Department of Physics, University of California San Diego, La Jolla CA 92093-0319.
}

(October 22, 2018)

\begin{abstract}
This work presents a step furthering a new perspective of proactive control of the spin-exciton dynamics in the quantum limit. Laser manipulation of spin-polarized optical excitations in a semiconductor nanodot is used to control the spin dynamics of two interacting excitons. Shaping of femtosecond laser pulses keeps the quantum operation within the decoherence time. Computation of the fidelity of the operations and application to the complete solution of a basic quantum computing algorithm demonstrate in theory the feasibility of quantum control.
\end{abstract}

Experimental laser control of spin states of excitons has been demonstrated in ensembles of semiconductor quantum dots (QDs) [1]. In a single dot, ultrafast control of spin-excitons [2], and entanglement of the electron-hole complex [3] have been reported. Here, we present a study of the design of laser pulses for the ultrafast control of the spin dynamics of individual excitons in a QD. This is a special case of designing Hamiltonians to bring a system from one state to another [4]. We hope that the theory of control of exciton spin dynamics may help rapid realization of basic quantum operations in nanodots. An exciting application would be the further development of quantum computation 5] using excitons [6-10] or intersubband transitions 11 13.

In this paper, the control of exciton spin dynamics consists in designing laser pulses to create as a function of time desired multiexciton states of a subsystem of dots. In the ultrafast control of the spin dynamics of excitons, there is the possibility of unintended dynamics due to the presence of close resonances. Sharp resonant pulses can minimize such unintended dynamics, but at the cost of a long operation time. The latter leads to the spontaneous recombination of the excitons and to their dephasing, resulting in uncontrolled deterioration of the amplitude and phase of the coefficients of the desired linear combination of quantum states. We give a solution to these two contradictory requirements by suggesting extending the wellknown laser pulse-shaping technique [14] to the delicate control in the quantum limit. Design of excitations has been applied to quantum computation by NMR [15] and by electron spin dynamics or intersubband transitions in QDs [16 19. We rely on the theory of DiVincenzo 20 stating that quantum gates operating on just two qubits at a time are sufficient to construct a general quantum circuit in a system of many dots. As an application to a prototype quantum computation which illustrates the issues raised above, we present the results of numerical simulations of the two exciton dynamics in a sequence of operations to solve the Deutsch-Jozsa (DJ) problem 21, 5. We shall concentrate on the control of the lowest four states formed by two excitons with opposite spins in a single dot. The spin-down and up states can be excited by the left $(\sigma-)$ and right-handed $(\sigma+)$ circularly polarized light, respectively. The four basis states are, in order: the lowest biexciton state $|+-\rangle$, the $\sigma+$ exciton state $|+\rangle$, the $\sigma-$ exciton state $|-\rangle$, and the ground state $|0\rangle$. We use the interaction representation for the states. We allow for the presence of other multiexciton states in the dot and the connection of the dot to the environment.

For precise control of coherent exciton dynamics, the probabilistic photo-excitation process of an exciton needs to be replaced by a resonant Rabi rotation which can create a linear combination of two states at a definite time. The primary condition for carrying out a Rabi rotation is sufficient light intensity without generating more excitons. This can be ascertained by analyzing the coherent nonlinear optical spectra. The starting point of our calculation is the energies and wave functions of the multiexciton states in a dot. For the current purpose, it is sufficient to obtain these states from two confined levels of electrons and holes each in a parallelepiped QD [22]. The size of the dot, $35 \mathrm{~nm} \times 40 \mathrm{~nm} \times 5 \mathrm{~nm}$, is typical of interface fluctuation quantum dots [23. Only Coulomb interaction between the carriers which conserves their conduction or valence band indices is taken into account exactly. The resulting states are used to compute the nonlinear spectra in a coherent pump and probe experiment setup as in 23. Each cw light beam has a narrow frequency peak and a definite circular polarization. The two beams have a fixed phase difference. The nonlinear spectrum is the absorption of the probe beam as a function of its frequency for a given intensity and frequency of the pump beam. An example of the calculated spectrum is shown in Fig. 1 for the cross-polarized circular configuration. The peaks come from transitions between the multi-exciton states. The key to our quantum operations is the lowest two peaks, corresponding to the exciton-biexciton and the ground state-exciton transitions, respectively (magnified in the inset). The separation of $1 \mathrm{meV}$ between the two peaks corresponds to the binding energy of $|+-\rangle$. An exciton lifetime of $40 \mathrm{ps}$ is taken from the experiment [23. The doublet structure in each peak is the Rabi split- 
ting due to the presence of the strong pump field. The observation in the nonlinear spectrum of these doublets would indicate the feasibility of Rabi rotations in realtime operations. The life-time broadening and the line positions yield the time limit on the pulses to be used in the exciton operations. The observation of Rabi rotations in QDs has not yet been reported. Evidence of Rabi flopping for excitons in semiconductor quantum wells 24] is encouraging.

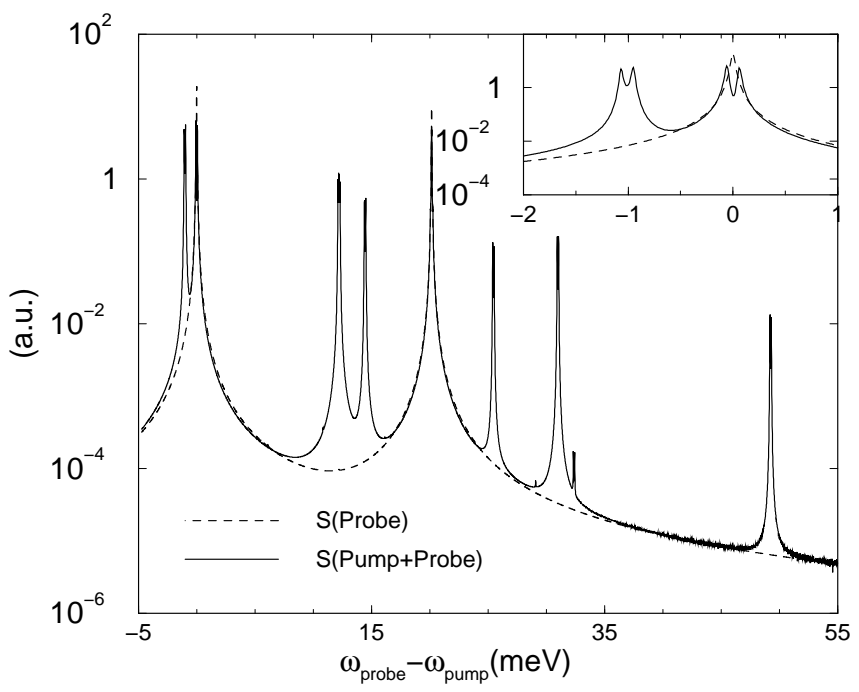

FIG. 1. Coherent nonlinear spectra of a QD with pump and probe in opposite circular polarization. The pump is resonant with the lowest excitonic state. The solid line is for the pump beam with intensity corresponding to a Rabi energy of $50 \mu \mathrm{eV}$ and the dashed line for zero pump intensity. The inset is a zoom on the first two levels.

The first quantum operation considered here is the conditional dynamics where the $\sigma$ - exciton will be given a $\pi$ rotation only if there is already a $\sigma+$ exciton present. This controlled Rabi rotation (C-ROT) is related to the controlled-not (C-NOT) or exclusive-or logic operation between two qubits [5]. A C-NOT operation inverts the second qubit (the target) depending on the state of the first qubit (the control). The C-ROT operation is accomplished simply by using a $\sigma-$ circularly polarized laser pulse to effect a single resonant Rabi $\pi$-rotation [25, 11, 8] which exchanges the exciton state $|+\rangle$ with the biexciton state $-|+-\rangle$ [see Fig. 2(a)]. Ideally, the same pulse does not cause the transition between $|0\rangle$ and $|-\rangle$ because the interaction between two excitons cause this transition to be off-resonance by an amount equal to the binding energy of the biexciton. The second operation is a single qubit operation: it acts on an exciton regardless of the state of the other exciton. It requires a resonant Rabi rotation for each of the many transitions involving the exciton. In the 2-exciton subspace, an operation on, say, the $\sigma-$ exciton requires two frequencies for the two transitions between the ground state $|0\rangle$ and $|-\rangle$ and between $|+\rangle$ and $|+-\rangle$, i.e., a two-color laser pulse is needed. The idea of a resonant Rabi rotation for C-NOT was proposed by Lloyd [25] and by Barenco et al, [11]. To complete the C-NOT, we could add to a C-ROT additional operations. However, we have found no need in quantum algorithms to take this time consuming extra step. Proper rotations represent the natural choice for the physical design of algorithms using Rabi flopping. We will show below how C-NOT and Hadamard gates in the standard implementation of the DJ algorithm [5] can be replaced by C-ROT and $\pi / 2$ operations. Using proper rotations, we have also designed the optical control for quantum Fourier transform, which is key to several algorithms including factorization, and carried out the simulation 26].

We have simulated these two basic operations and calculated the gate fidelity defined in Ref. 27], whose equally weighted average over all the possible initial states gives $\frac{1}{10} \sum_{i}\left|I_{i i}\right|^{2}+\frac{1}{20} \sum_{i \neq j}\left(I_{i i}^{*} I_{j j}+I_{i j}^{*} I_{i j}\right) . I_{i j}=$ $\left\langle i\left|\bar{U}^{\dagger} U\right| j\right\rangle$, where $\bar{U}$ and $U$ are the calculated and the ideal quantum transformations respectively. We have included in addition to the four states in the computational basis the two copolarized biexciton states $|++\rangle,|--\rangle$ in order to check their contribution to the unintended dynamics. The other states are more distant in energy and can be neglected. The dephasing is taken into account phenomenologically as a stochastic process using the Monte Carlo wavefunction method 28]. A 40 ps exciton lifetime is taken from 23. If we use a spectrally sharp pulse to perform C-ROT, without dephasing the unintended perturbation is negligible but the operation time is of the same order as the dephasing time. On the other hand, a sufficiently short pulse (about $2 \mathrm{ps}$ ) to limit dephasing destroys the fidelity by unintended dynamics. We propose a remedy by pulse shaping. Let us first compare the results in fidelity of the operations before presenting the details of shaping. The fidelity decreases with increasing Rabi frequency. The calculated results used $2 \mathrm{meV}$ Rabi energy which is larger than the separation between the exciton and biexciton resonances of $1 \mathrm{meV}$. The fidelity for C-ROT is $0.535,0.966$, and 0.903 for, in order, unshaped short pulse (without dephasing), shaped pulse without and with dephasing. For single qubit $\pi / 2$ rotation, it is $0.678,0.995$ and 0.981 respectively. The fidelity is nicely restored by pulse shaping. The leakage to the unwanted states $|++\rangle,|--\rangle$ is less than $1 \%$.

In a C-ROT, instead of a spectrally narrow $\pi$ pulse resonant with the exciton-biexciton transition, we use a combination of two phase-locked pulses of the $\sigma$ - polarized field,

$$
\mathcal{E}(t)=\mathcal{E}_{0}\left(e^{-\left(t / t_{1}\right)^{2}-i \epsilon_{-} t}+e^{-\left(t / t_{2}\right)^{2}-i\left(\epsilon_{--} \Delta_{+-}\right) t+i \pi}\right) .
$$

For convenience, we use equal amplitudes. The energy of each state is denoted by $\epsilon$ with an index for that state. The biexciton binding energy is $\Delta_{+-}=\epsilon_{+}+\epsilon_{-}-\epsilon_{+-}$. The dynamics of the four exciton levels is shown in Fig. 2, visualized as pseudospin (Bloch vector) for the 
$\sigma$ - exciton in the absence or presence of the $\sigma+$ exciton (see insert (a)). In the absence of $\sigma+$, the $\sigma-$ exciton pseudospin evolves in an effective magnetic field $\mathbf{B}_{X}(t)=\mathbf{B}_{F}\left(t, t_{1}\right)+\mathbf{B}_{R}\left(t, t_{2}\right)$, composed of a component $\mathbf{B}_{F}$ fixed in the $x$-direction due to the resonant pulse with the $|0\rangle \leftrightarrow|-\rangle$ transition and another $\mathbf{B}_{R}$, due to the off-resonant pulse with the $|+\rangle \leftrightarrow|+-\rangle$ transition, which rotates clockwise in the $x y$-plane at the beat frequency $\Delta_{+-}$. In the presence of the $\sigma+$, the pseudospin is driven by an effective $\mathbf{B}_{X X}(t)=\mathbf{B}_{R}\left(t, t_{1}\right)+\mathbf{B}_{F}\left(t, t_{2}\right)$, in a rotating frame where the field component resonant with the biexciton resonance is fixed and the exciton field rotates anti-clockwise. The central idea is to optimize the control parameters $\left(t_{1}, t_{2}\right)$ in such a way that, at the end of the shaped pulse, $\mathbf{B}_{X X}$ produces a $\pi$ rotation for the biexciton resonance, and $\mathbf{B}_{X}$ brings the exciton pseudospin back to the original state. We have studied numerically the dynamics of the four levels in the control manifold generated by the parameters $\left(t_{1}, t_{2}\right)$. Even using Rabi energies larger than the biexcitonic binding energy, it is possible to find a region in the phase space $\left(t_{1}, t_{2}\right)$ fulfilling the required conditions. This is illustrated in Fig. 2(b), where the evolution of the pseudospin in the absence and presence of the + exciton using a pulse shaped as in Eq. (11), with $t_{1}=0.56 \mathrm{ps}$ and $t_{2}=1.05 \mathrm{ps}$, is represented on the Bloch sphere: at the end of the shaped pulse we have a $\pi$ rotation for $|+\rangle \leftrightarrow|+-\rangle$, while $|0\rangle$ remains unaffected. As inferred from the calculation of the fidelity, this shaping works for an arbitrary initial state, and the pulse acts as a general rotor. A simple intuitive picture is given by the frequency spectrum of the pulse in Eq.(11), shown in Fig. 2(c). The two phase-locked components have opposite phases and the chosen $t_{1}$ and $t_{2}$ control the interference in the electric field giving a zero amplitude at the exciton resonance and a finite value at the biexciton resonance. The ultrafast manipulation of the qubits is carried out using light interference. For the $\pi / 2$ single qubit gate we used $t_{1}=t_{2}=0.15 \mathrm{ps}$ and inphase components. The full theory for the application of the shaping to more general operations and limitations will be provided elsewhere [26.

A cogent illustration of the time limitation of decoherence and the designs to beat the limit is given by a numerical simulation of a complete quantum computing algorithm. We choose the two-qubit DJ problem 21] both for its simplicity and for its fundamental nature. It has been implemented by nuclear magnetic resonance (NMR) [29]. For a single qubit variable $x$, there are four possible single-qubit functions: $f_{1}(x)=0$ and $f_{2}(x)=1$ belong to the class of constant functions and $f_{3}(x)=x$ and $f_{4}(x)=1-x$ the class of balanced functions. The DJ problem is to determine whether a given function is constant or balanced. Classically, the only way to find out if the function is constant or balanced is to calculate the function for both the 0 and 1 input values and then compare the results. The quantum algorithm de- signs a unitary transformation which parallel-processes all possible values of $x$. We use the presence or absence of $\sigma \pm$ excitons to represent the two qubits. The complete solution consists of three steps: (i) Encoding transforms the initial ground state dot into an input state: $|i n\rangle=\frac{1}{2}(-|+-\rangle+|+\rangle-|-\rangle+|0\rangle)$ by the single-qubit $\sigma+-\pi / 2$ rotation and $\sigma-\pi / 2$ rotation. (ii) The unitary transformation $U_{f_{j}}|y, x\rangle=R_{x}(\pi)^{1-2 f_{j}(y)}|y, x\rangle$, associated to the given $f_{j}$ is applied.This is a conditional rotation acting on qubit $x$ ( $\sigma$ - exciton), conditioned on the effect of $f_{j}$ on the qubit $y$ ( $\sigma+$ exciton). (iii) $D e-$ coding by $\pi / 2$ rotations of both circular polarizations, ending with the ground state if the function is a constant and the $|+\rangle$ state if the function is balanced. The final measurement on the $\sigma+$ exciton can be made with the cross-polarization probe or with the emitted photon polarization.
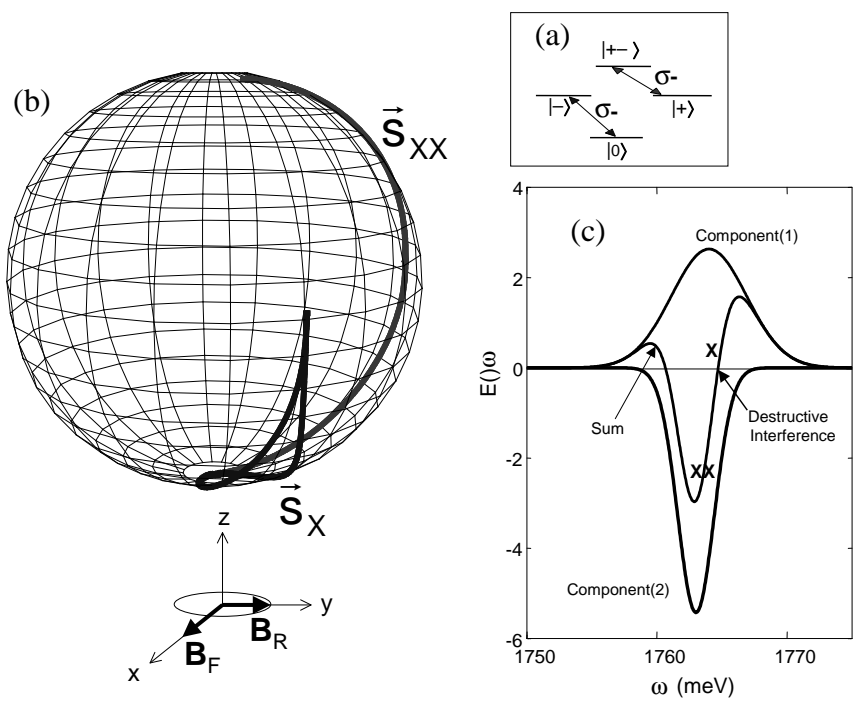

FIG. 2. Dynamics of spectral shaping. (a) Energy schematics for the transitions generated by a $\sigma-$ pulse. (b) Evolution of the exciton $\vec{s}_{X}$ and biexciton $\vec{s}_{X X}$ pseudospins on the Bloch sphere under a shaped pulse. (c) Fourier transform of the shaped pulse and its components.

The evolution of the qubits through the DJ algorithm is shown in Fig. 3 as the real parts of the coefficients of the corresponding states in two rows for a constant $\left(f_{2}\right)$ and a balanced $\left(f_{3}\right)$ function. The first column depicts the ideal case of sharp resonance functions without dephasing as described above. Even with the maximum packing of the pulses (using overlap at $10 \%$ of the peak electric field without substantial deterioration of the results), the first column show the time to complete the quantum computation above the adopted decoherence time of the order of $40 \mathrm{ps}$. Simple reduction of the pulse time to within dephasing time while keeping the pulse area leads to a failure to discriminate the solution, as shown in column 2. Shrinking the temporal width of the pulses causes a 
loss of the spectral selectivity needed to distinguish between the excitonic and biexciton resonances. In order to exhibit the effects of the unintended dynamics and of the pulse-shaping remedy, the first three column have not included decoherence. The effect of shaping on quantum computation is shown the third column [30]: the possibility to distinguish between constant and balanced within the decoherence time is recovered.
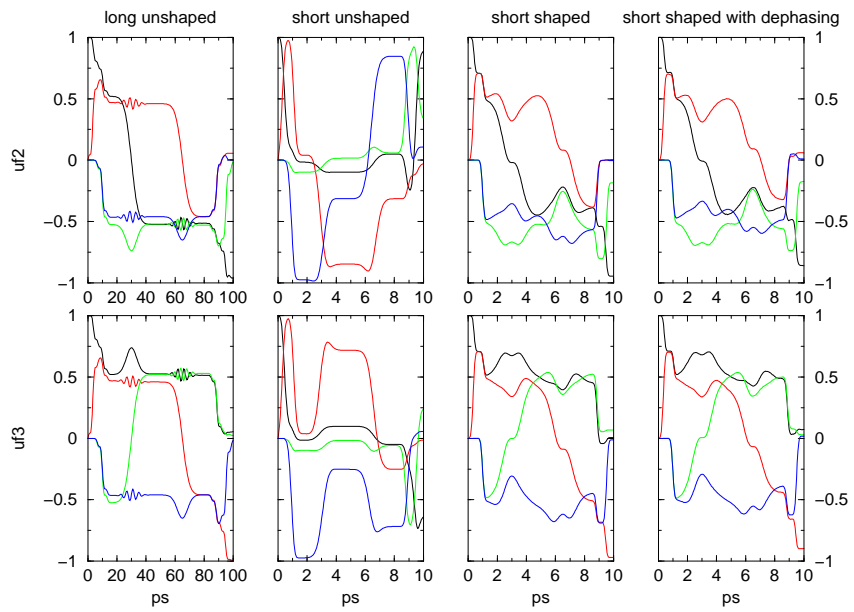

FIG. 3. Evolution of the qubits during a quantum computation. The real parts of the coefficients $\mathcal{R} c_{j}(t)$ for the first four levels are plotted. See text for explanation.

The robustness of our scheme is tested with the inclusion of decoherence in column 4. The pulse shaping can in principle be applied to more than two excitons. There is still the question of whether the pulse shaping is "scalable" to a system of $n$ qubits, as $n$ increases. The advantage of a quantum computer over a classical one is the saving of effort from $2^{n}$ steps to order of powers in $n$ [5]. We need to avoid using an algorithm which treats the $n$ excitons locked together as a strongly interacting system. The method [20] is to process in steps, each involving a few excitons. Then, the overhead of shaping is at most a few powers of $n$ 18, i.e., it does not spoil the exponential saving.

In conclusion, we resolve the basic physics issues raised by the optical control of two antiparallel-spin excitons in a quantum dot. The application of this idea to the control of two spin-polarized excitons in a single dot is well within present experimental capabilities, and we hope that our explicit design will stimulate a quick realization of an elementary quantum control. Our solution of pulseshaping is a special case of quantum control of interacting spins and may be applied to other physical systems, such as trapped ions. Future work would involve excitons in different dots. Several schemes already exist for scalable system where qubits stored in an array of dots can be swapped around in a distributed quantum computer [6.7]. Cavity Quantum Electrodynamics effects can be used for the interconnection between dots, and have, in principle, the additional potential of controlling the spontaneous emission of the excitons.

LJS thanks Drs. D. Steel, I. Chuang, D. Gammon, and Hailin Wang for helpful discussions. CP acknowledges discussions with Dr. J. Fernandez-Rossier and the support by the Swiss NSF. This work was supported in part by the NSF Grant No. DMR 9721444 and in part by DARPA/ONR N0014-99-1-109.

\section{APPENDIX}
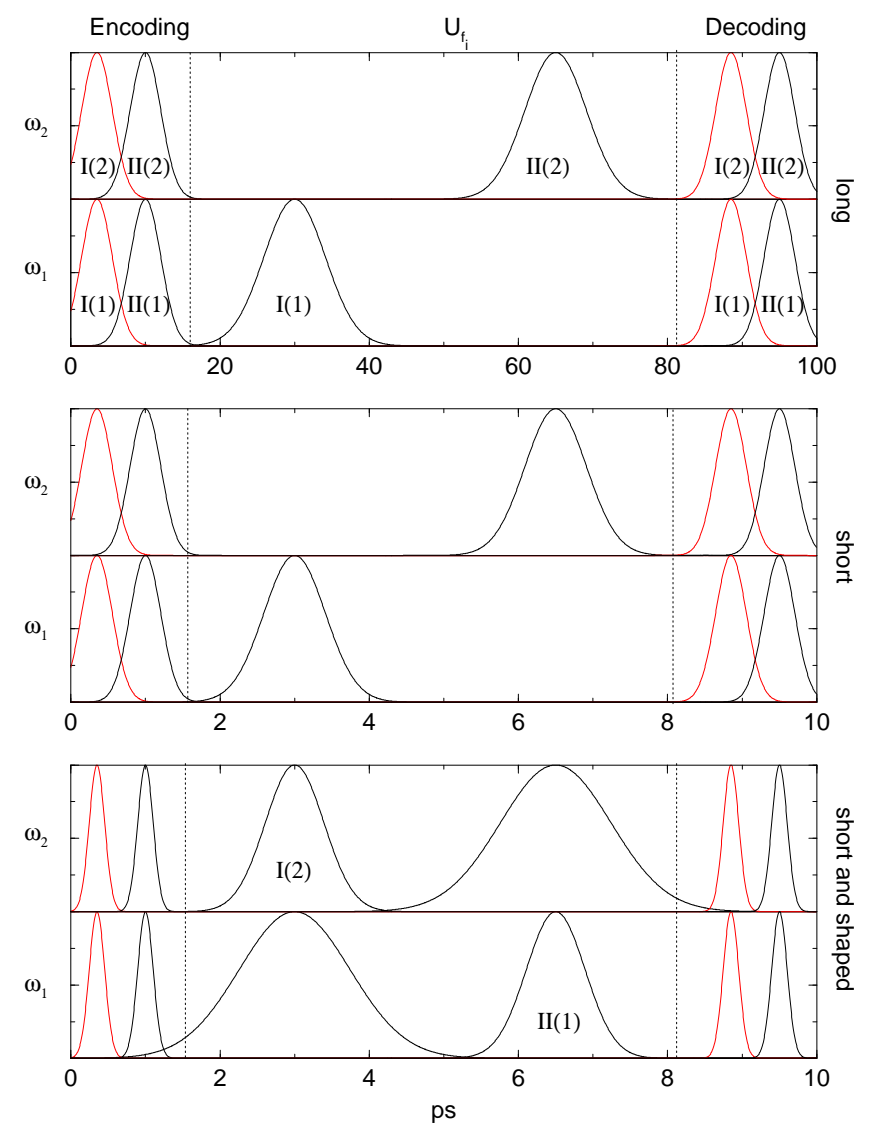

FIG. 4. Slowly varying envelopes of the pulse sequences used in the quantum computation for the solution of the DJ problem in Fig. 3. The red (black) line indicates $\sigma+(\sigma-)$ circular polarization. The two rows labeled by $\omega_{i}$ refers to the resonance frequency of the pulses: $\omega_{1}=\epsilon_{+}=\epsilon_{-}, \omega_{2}=\epsilon_{+}-\Delta_{+-}=\epsilon_{-}-\Delta_{+-}$. 
TABLE I. Pulse sequences used in the computation, (1) and (2) indicate the two components of a shaped pulse of the form $\mathcal{E}(t)=\mathcal{E}_{1}(t)+\mathcal{E}_{2}(t)$ $=\mathcal{E}_{0}\left(e^{-\left(t / t_{1}\right)^{2}-i \omega_{1} t-i \phi_{1}}+e^{-\left(t / t_{2}\right)^{2}-i \omega_{2} t+i \phi_{2}}\right)$. The area $\theta$ is defined as $\hbar \theta=\int d \mathcal{E}(t) d t$. $d \mathcal{E}_{0}$ is $0.2 \mathrm{meV}$ in the long pulses case and $2 \mathrm{meV}$ for short and shaped pulses. The pulse sequence of $U_{f_{1}}\left(U_{f_{4}}\right)$ is identical to the $U_{f_{2}}\left(U_{f_{3}}\right)$ except for a change of sign in the phase $\phi_{j}$.

\begin{tabular}{|c|c|c|c|c|c|c|}
\hline & $\mathrm{Pol}$ & $\phi_{j}$ & $\omega_{j} \mid$ & $\begin{array}{c}\text { Long pulses } \\
\text { ps }(\theta)\end{array}$ & $\begin{array}{c}\text { Short pulses } \\
\text { ps }(\theta)\end{array}$ & $\begin{array}{c}\text { Shaped pulses } \\
\operatorname{ps}(\theta)\end{array}$ \\
\hline \multicolumn{7}{|c|}{ ENCODING } \\
\hline$\overline{\mathrm{I}(1)}$ & $\sigma+$ & $-\pi / 2$ & $\omega_{1}$ & $2.92(\pi / 2)$ & $0.29(\pi / 2)$ & $0.15(0.81)$ \\
\hline I $(2)$ & $\sigma+$ & $-\pi / 2$ & $\omega_{2}$ & $2.92(\pi / 2)$ & $0.29(\pi / 2)$ & $0.15(0.81)$ \\
\hline II (1) & $\sigma-$ & $\pi / 2$ & $\omega_{1}$ & $2.92(\pi / 2)$ & $0.29(\pi / 2)$ & $0.15(0.81)$ \\
\hline II (2) & $\sigma-$ & $\pi / 2$ & $\omega_{2}$ & $2.92(\pi / 2)$ & $0.29(\pi / 2)$ & $0.15(0.81)$ \\
\hline \multicolumn{7}{|c|}{ DECODING } \\
\hline$\overline{\mathrm{I}(1)}$ & $\sigma+$ & $\overline{\pi / 2}$ & $\omega_{1}$ & $2.92(\pi / 2)$ & $0.29(\pi / 2)$ & 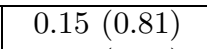 \\
\hline I $(2)$ & $\sigma+$ & $\pi / 2$ & $\omega_{2}$ & $2.92(\pi / 2)$ & $0.29(\pi / 2)$ & $0.15(0.81)$ \\
\hline II (1) & $\sigma-$ & $\pi / 2$ & $\omega_{1}$ & $2.92(\pi / 2)$ & $0.29(\pi / 2)$ & $0.15(0.81)$ \\
\hline$\underline{\mathrm{II}}(2)$ & $\sigma-$ & $\pi / 2$ & $\omega_{2}$ & $2.92(\pi / 2)$ & $0.29(\pi / 2)$ & $0.15(0.81)$ \\
\hline \multicolumn{7}{|c|}{$U_{f_{2}}$} \\
\hline$\overline{\mathrm{I}(1)}$ & $\sigma-$ & $-\pi / 2$ & $\omega_{1}$ & $5.83(\pi)$ & $0.58(\pi)$ & $1.05(5.68)$ \\
\hline I $(2)$ & $\sigma-$ & $\pi / 2$ & $\omega_{2}$ & & & $0.56(3.03)$ \\
\hline $\mathrm{II}(2)$ & $\sigma-$ & $-\pi / 2$ & $\omega_{2}$ & $5.83(\pi)$ & $0.58(\pi)$ & $1.05(5.68)$ \\
\hline$\underline{\mathrm{II}(1)}$ & $\sigma-$ & $\pi / 2$ & $\omega_{1}$ & & & $0.56(3.03)$ \\
\hline \multicolumn{7}{|c|}{$U_{f_{3}}$} \\
\hline I (1) & $\sigma-$ & $-\pi / 2$ & $\omega_{1}$ & $5.83(\pi)$ & $0.58(\pi)$ & $1.05(5.68)$ \\
\hline I $(2)$ & $\sigma-$ & $\pi / 2$ & $\omega_{2}$ & & & $0.56(3.03)$ \\
\hline $\mathrm{II}(2)$ & $\sigma-$ & $\pi / 2$ & $\omega_{2}$ & $5.83(\pi)$ & $0.58(\pi)$ & $1.05(5.68)$ \\
\hline $\mathrm{II}(1)$ & $\sigma-$ & $-\pi / 2$ & $\omega_{1} \mid$ & & & $0.56(3.03)$ \\
\hline
\end{tabular}

[1] J.A. Gupta, D.D. Awschalom, X. Peng, and A.P. Alivisatos, Phys. Rev. B 59, 10421 (1999).

[2] N.H. Bonadeo et al., Science 282, 1473 (1998).

[3] G. Chen et al., Science 289, 1906 (2000).

[4] W. S. Warren, H. Rabitz, M. Dahleh, Science 259, 1581 (1993).

[5] M.A. Nielsen and I. Chuang, Quantum Computation and Quantum Information (Cambridge University Press, Cambridge, 2000).

[6] A. Imamoglu et al. Phys. Rev. Lett. 83, 4204 (1999).

[7] T. A. Brun, H. Wang, Phys. Rev. A 61, 032307 (2000).

[8] F. Troiani, U. Hohenester, E. Molinari, Phys. Rev. B 62, R2263 (2000).

[9] E. Biolatti, R. C. Iotti, P. Zanardi, F. Rossi, Phys. Rev. Lett. 85, 5647 (2000).

[10] P. Chen, C. Piermarocchi, and L.J. Sham, Physica E, to be published, preprint in cond-mat/0009307.

[11] A. Barenco, D. Deutsch, A. Ekert, R. Jozsa, Phys. Rev. Lett. 74, 4083 (1995).

[12] G. D. Sanders, K. W. Kim, W. C. Holton, Phys. Rev. B 61, 7526(2000).

[13] M.S. Sherwin, A. Imamoglu and T. Montroy, Phys. Rev. A 60, 3508 (1999).

[14] A.M. Weiner, Prog. Quant. Electr. 19, 161 (1995).

[15] H. K. Cummins, J. A. Jones, New J. of Phys. 2, 6.1-6.12 (2000).

[16] G. P. Berman, D.K. Campbell, V.I. Tsifrinovich, Phys. Rev. B 55, 5929 (1997).

[17] G. Burkard, D. Loss, D. P. Di Vincenzo, J. A. Smolin, Phys. Rev. B 60, 11404 (1999).

[18] L. Tian, S. Lloyd, Phys. Rev. A 62, 050301 (2000).

[19] G. D. Sanders, K. W. Kim, W. C. Holton, Phys. Rev. B 59, 1098(1999).

[20] D.P. DiVincenzo, Phys. Rev A 51, 1015 (1995).

[21] D. Deutsch, R. Jozsa, Proc. Roy. Soc. London A 439, 553 (1992).

[22] A. Barenco, M. A. Dupertuis, Phys. Rev. B 52, 2766 (1995).

[23] N. H. Bonadeo et al. Phys. Rev. Lett. 81, 2759 (1998).

[24] A. Schülzgen et al., Phys. Rev. Lett. 82, 2346 (1999); F. Quochi et al., ibidem 80, 4733 (1998).

[25] S. Lloyd, Science 261, 1569 (1993).

[26] P. Chen, C. Piermarocchi, and L. J. Sham, to be published.

[27] J.F. Poyatos, J.I. Cirac, and P. Zoller, Phys. Rev. Lett. 78, 390 (1997).

[28] K. Mølmer, Y. Castin, J. Dalibard, J. Opt. Soc. Am B 10, 524 (1993); R. Schack and T. A. Brun, Comp. Phys. Commun. 102, 210 (1997).

[29] I. L. Chuang, L. M. K. Vandersypen, X. Zhou, D. W. Leung, S. Lloyd, Nature 393, 143 (1998); J. A. Jones, M. Mosca, J. Chem. Phys. 109, 1648 (1998).

[30] Details of pulse sequences are given in the Appendix. 